\title{
ON GRUNDY TOTAL DOMINATION NUMBER IN PRODUCT GRAPHS
}

\author{
BOŠTJAN BREŠAR \\ Faculty of Natural Sciences and Mathematics, University of Maribor, Slovenia \\ Institute of Mathematics, Physics and Mechanics, Ljubljana, Slovenia \\ e-mail: bostjan.bresar@um.si \\ Csilla Bujtás
}

Faculty of Information Technology, University of Pannonia, Veszprém, Hungary e-mail: bujtas@dcs.uni-pannon.hu

\section{TAnja GologranC}

Faculty of Natural Sciences and Mathematics, University of Maribor, Slovenia Institute of Mathematics, Physics and Mechanics, Ljubljana, Slovenia

e-mail: tanja.gologranc@gmail.com

\section{SANDI KLAVŽAR}

Faculty of Mathematics and Physics, University of Ljubljana, Slovenia Faculty of Natural Sciences and Mathematics, University of Maribor, Slovenia Institute of Mathematics, Physics and Mechanics, Ljubljana, Slovenia e-mail: sandi.klavzar@fmf.uni-lj.si

\section{GAŠPER KoŠMRLJ}

Institute of Mathematics, Physics and Mechanics, Ljubljana, Slovenia Abelium R\&D, Ljubljana, Slovenia

e-mail: gasperk@abelium.eu

Tilen Marc

Institute of Mathematics, Physics and Mechanics, Ljubljana, Slovenia Faculty of Mathematics and Physics, University of Ljubljana, Slovenia e-mail: marct15@gmail.com 


\title{
BALÁzs PATKós
}

Alfréd Rényi Institute of Mathematics, Hungarian Academy of Sciences

Budapest, Hungary

e-mail: patkos@renyi.hu

\section{Zsolt TuZA}

Faculty of Information Technology, University of Pannonia, Veszprém, Hungary Alfréd Rényi Institute of Mathematics, Hungarian Academy of Sciences

Budapest, Hungary

e-mail: tuza@dcs.uni-pannon.hu

AND

MÁTÉ ViZeR

Alfréd Rényi Institute of Mathematics, Hungarian Academy of Sciences Budapest, Hungary

e-mail: vizermate@gmail.com

\begin{abstract}
A longest sequence $\left(v_{1}, \ldots, v_{k}\right)$ of vertices of a graph $G$ is a Grundy total dominating sequence of $G$ if for all $i, N\left(v_{i}\right) \backslash \bigcup_{j=1}^{i-1} N\left(v_{j}\right) \neq \emptyset$. The length $k$ of the sequence is called the Grundy total domination number of $G$ and denoted $\gamma_{g r}^{t}(G)$. In this paper, the Grundy total domination number is studied on four standard graph products. For the direct product we show that $\gamma_{g r}^{t}(G \times H) \geq \gamma_{g r}^{t}(G) \gamma_{g r}^{t}(H)$, conjecture that the equality always holds, and prove the conjecture in several special cases. For the lexicographic product we express $\gamma_{g r}^{t}(G \circ H)$ in terms of related invariant of the factors and find some explicit formulas for it. For the strong product, lower bounds on $\gamma_{g r}^{t}(G \otimes H)$ are proved as well as upper bounds for products of paths and cycles. For the Cartesian product we prove lower and upper bounds on the Grundy total domination number when factors are paths or cycles.
\end{abstract}

Keywords: total domination, Grundy total domination number, graph product.

2010 Mathematics Subject Classification: 05C69, 05C76.

Received 27 February 2018

Revised 26 September 2018

Accepted 26 September 2018 